\title{
Fulvestrant and trastuzumab in patients with luminal HER2-positive advanced breast cancer (ABC): an Italian real-world experience (HERMIONE 9)
}

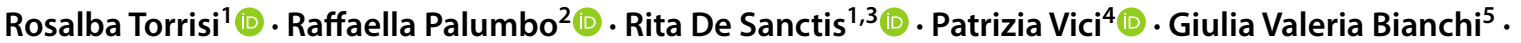 \\ Laura Cortesi $^{6}$. Vita Leonardi ${ }^{7}$ (D) $\cdot$ Rossana Gueli $^{8}$ (D) . Alessandra Fabi ${ }^{9}$ (D) . Maria Rosaria Valerio ${ }^{10}$ (D) . \\ Anna Rita Gambaro ${ }^{11}$ - Barbara Tagliaferri2 ${ }^{2}$ D $\cdot$ Laura Pizzuti $^{4} \cdot$ Marina Elena Cazzaniga $^{12}$ (D) Armando Santoro ${ }^{1,3}$ (iD
}

Received: 11 May 2021 / Accepted: 20 August 2021

(c) The Author(s), under exclusive licence to Springer Science+Business Media, LLC, part of Springer Nature 2021

\begin{abstract}
Purpose The most appropriate therapy for HR +/HER2-positive (HER2 +) advanced breast cancer (ABC) is a matter of debate. Co-targeting of both receptors represents an attractive strategy to overcome the cross-talk between them.

Methods The HERMIONE 9 is an observational retrospective multicentric study which aimed to describe the clinical outcome of patients with HR +/HER2 + ABC who received the combination of Fulvestrant (F) and Trastuzumab (T) as part of their routine treatment at 10 Italian Institutions.

Results Eighty-seven patients were included. Median age was 63 (range, 35-87) years. The median number of previous treatments was 3 (range, $0-10$ ) and $\mathrm{F}$ and $\mathrm{T}$ were administered as $\geq 3 \mathrm{rd}$ line in 67 patients. Among the 86 evaluable patients, $6(6.9 \%)$ achieved CR, 18 (20.7\%) PR, and 44 (50.6\%) had SD $\geq 24$ weeks with an overall CBR of 78.2\%. At a median follow-up of 33.6 months, mPFS of the entire cohort was 12.9 months (range, 2.47-128.67). No difference was observed in mPFS between patients treated after progression or as maintenance therapy (mPFS 12.9 and 13.9 months in 64 and 23 patients, respectively), neither considering the number of previous treatment lines $(\leq 3$ or $<3)$.

Conclusion The combination of $\mathrm{F}$ and $\mathrm{T}$ was active in this cohort at poor prognosis and deserves further investigations possibly in combination with pertuzumab in patients with high ER expression.
\end{abstract}

Keywords Trastuzumab $\cdot$ Fulvestrant $\cdot$ Hormone receptor positive $\cdot$ HER2 positive $\cdot$ Advanced breast cancer

Rosalba Torrisi

rosalba.torrisi@humanitas.it

1 Department of Medical Oncology and Hematology Unit, IRCCS Humanitas Research Hospital, via A. Manzoni 56 20089, Rozzano, Milano, Italy

2 Oncologia Medica, IRCCS ICS Maugeri, Pavia, Italy

3 Department of Biomedical Sciences, Humanitas University, Pieve Emanuele, Italy

4 UOC OM2 IRCCS Regina Elena National Cancer Institute, Roma, Italy

5 SC Oncologia Medica 1 Fondazione IRCCS Istituto Nazionale Tumori, Milano, Italy

6 Department of Oncology and Haematology, Modena Hospital University, Modena, Italy
7 Department of Medical Oncology, ARNAS Civico, Palermo, Italy

8 Medical Oncology, ASST Settelaghi, Varese, Italy

9 Precision Medicine in Breast Cancer Unit, Scientific Directorate, Fondazione Policlinico Universitario A. Gemelli, IRCCS, Rome, Italy

10 Medical Oncology Unit, Department of Surgical, Oncological and Oral Sciences, University of Palermo, Palermo, Italy

11 Medical Oncology, ASST Fatebenefratelli Sacco PO Sacco, Milano, Italy

12 Centro Ricerca Fase 1 ASST Monza and Università Degli Studi Milano Bicocca, Milano, Italy 


\section{Introduction}

Gene expression studies have clearly demonstrated in recent years that HER2-positive (HER2 +) breast cancer is a heterogeneous disease [1]. Approximately, 50\% of HER 2 + tumors are hormone receptor positive $(\mathrm{HR}+)$ at immunohistochemistry (IHC) [2]

However, only one half of HER2 + tumors at IHC correspond to the HER2-enriched subtype as defined by gene expression profiling, and within the latter subtype significant molecular differences were observed according to the HR expression [3]

However, these immunohistochemical and molecular differences do not translate in different therapeutic strategies for treatment of HR +/HER2 + tumors which routinely include chemotherapy and anti-HER2 agents [4]. Preclinical evidence suggesting that HER2 overexpression was associated with endocrine resistance [5], further confirmed by the poor results of earlier studies investigating a chemotherapy-free regimen in advanced $\mathrm{HR}+/ \mathrm{HER} 2+$ tumors $[6,7]$, supported an unique treatment strategy including chemotherapy plus anti-HER 2 therapy irrespective of HR status [5]. However, results from neoadjuvant trials and from the advanced setting clearly indicated less sensitiveness of luminal HER2 + tumors to this approach probably due to a bidirectional cross-talk between the two driver receptors, estrogen and HER2 [8-11]. Co-targeting of both receptors appears thus an attractive strategy to overcome the resistance due to this cross-talk [12].

The availability of fulvestrant which internalizes ER after binding, leading to a more complete abolishment of the estrogen signaling with respect to aromatase inhibitors, might represent an attractive targeted partner for a full HR/ HER2 blockade [13]. A synergistic effect between fulvestrant and trastuzumab in inhibiting growth in cell lines and xenografts has been shown, but only a few in vivo data are available on the combination [14]

In the present retrospective observational study we report a real-world series of patients with $\mathrm{HR}+$ I HER2 + advanced breast cancer (ABC) treated with the combination of fulvestrant and trastuzumab and analyze possible predictive factors of its effectiveness.

\section{Patients and methods}

The HERMIONE 9 is an observational retrospective multicentric study which aimed to describe the clinical outcome of patients with HR +/HER2 + ABC who received the combination of fulvestrant and trastuzumab as part of their routine treatment at 10 Italian Institutions participating to the HERMIONE platform.

Eligible patients were pre- and postmenopausal women aged $\geq 18$ years diagnosed with $\mathrm{HR}+$ (defined as ER and/ or PgR $\geq 10 \%$ ) and HER 2 + (defined as IHC $3+$ or FISH or CISH or SISH positive) inoperable locally advanced and/or metastatic breast cancer. Premenopausal patients received concomitant ovarian suppression with GnRH analogues. Fulvestrant + trastuzumab were administered as any line of treatment. Fulvestrant was administered $500 \mathrm{mg}$ i.m. q28 days with loading dose after 14 days; trastuzumab was administered q21 days either as $8 \mathrm{mg} / \mathrm{kg}$ loading dose and then $6 \mathrm{mg} / \mathrm{kg}$ i.v. infusion or $600 \mathrm{mg}$ s.c., according to the internal institutional guidelines.

Patient medical charts were reviewed and tumor response was assessed. Since this is a retrospective study response could not be evaluated according to RECIST 1.1 criteria in all patients, but CT scans and other imaging were reviewed in order to comply with RECIST criteria whenever possible.

Information on age at diagnosis, menopausal status, previous adjuvant therapies, number of previous therapies for metastatic disease, number and response to previous therapies for metastatic disease, pathological features of primary and metastatic tumor if available, sites of metastatic disease, best response, and disease progression if occurred, and last follow-up visit were collected.

This study was approved by the institutional ethical committee of the coordinating center (Humanitas Research Hospital) and of all the other participating Institutions and was conducted in compliance with Helsinki Declaration.

\section{Statistical analysis}

Clinical data were summarized as frequencies and proportions or as medians and range. To assess the tumor heterogeneity, we calculated the agreement in the tumor biological profile between primary and metastatic sites in terms of ER, PgR, and HER2 expression using Cohen's K statistics. Response to treatment was classified as complete response $(\mathrm{CR})$, partial response (PR), stable disease (SD), or progressive disease (PD) according to the Response Evaluation Criteria in Solid Tumors (RECIST) criteria version 1.1 whenever possible. Clinical Benefit Rate (CBR) was defined as the percentage of patients who achieved CR, PR, and $\mathrm{SD} \geq 24$ weeks. Survival curves were estimated using the Kaplan-Meier method. Progression-Free Survival (PFS) was defined as the time from the first day of fulvestrant + trastuzumab until disease progression, as shown by radiological or clinical examination, or death from any cause. Patients without any evidence of progressive disease were censored at the date of their last follow-up. PFS curves were analyzed according to disease status at fulvestrant + trastuzumab start, early versus later fulvestrant + trastuzumab line of treatment, 
the type of previous therapy, and HR status. Differences among subgroups were evaluated using the log-rank test.

Statistical significance was set at 0.05 . Analyses were performed with the STATA software package, version 15 and with STATISTICA version 7, StatSoft, Inc.

\section{Results}

From August 2005 to March 2021 a total of 87 patients were treated with the combination of fulvestrant + trastuzumab at the 10 participating Institutions.

Median age was 63 years (range, 35-87). Clinical pathological patients' characteristics are reported in Table 1. Thirty-one percent (27/87) of the patients were diagnosed with de novo metastatic breast cancer; in recurrent $\mathrm{ABC}$ (60/87, 69\%), median time to metastatic disease was 57.1 months (range, 7.0-330.5).

A biopsy of metastatic site was available in 48 out of 87 patients (45.9\%); biological characteristics of metastases were concordant with those of primary tumors except for PgR expression which was significantly lower $(p=0.02)$ As a consequence, 59 patients $(67.8 \%)$ had triple-positive tumors as determined in the primary tumor while in metastatic biopsies only $22 / 45$ (49\%) metastatic tumors were triple positive.

The median number of previous treatments (both chemotherapy \pm trastuzumab and endocrine therapy + trastuzumab) for advanced disease was 3 (range, $0-10$ ). Fulvestrant + trastuzumab were administered as $\geq 3$ line in 67 patients. All patients had received trastuzumab previously while only $19 \%$ of patients had not received prior endocrine therapy for $\mathrm{ABC}$

Twenty-three patients started fulvestrant + trastuzumab as maintenance treatment while 64 patients had progressed upon the previous regimen (20 patients after endocrine therapy + trastuzumab, 22 after anti-HER 2 agents + chemotherapy, and 20 after chemotherapy).

The median duration of fulvestrant + trastuzumab treatment was 11.5 months (range, 2.03-128.67). Trastuzumab was administered i.v. $(57 / 87,65.52 \%)$, or s.c. $(15 / 87$, $17.24 \%)$, or both $(11 / 87,12.64 \%)$; the data were not available in 4 cases.

Eight-six patients were evaluable for response: 6 patients achieved CR (6.9\%), 18 patients $(20.7 \%)$ PR, and 51 patients $(58.6 \%) \mathrm{SD}$-of whom 44 patients $(50.6 \%)$ had $\mathrm{SD} \geq 24$ weeks. Overall, the CBR was $78.2 \%$. At a median follow-up of 33.6 months (range, 2.47-139.27), mPFS of the entire cohort was 12.9 months (range, 2.47-128.67; Fig. 1). A shorter time to progression (TTP) was observed during the treatment immediately preceding fulvestrant + trastuzumab (7.62 months, range, 2.0-98.9). No statistically significant difference was observed in mPFS in patients treated after
Table 1 Baseline patient and disease characteristics

\begin{tabular}{|c|c|}
\hline Median age, years (range) & $63(35-87)$ \\
\hline De novo mBC, n (\%) & $27(31.03)$ \\
\hline \multicolumn{2}{|l|}{ Menopausal status, $n(\%)$} \\
\hline Pre-menopausal & $24(27.59)$ \\
\hline Post-menopausal & $60(68.96)$ \\
\hline Unknown & $3(3.45)$ \\
\hline \multicolumn{2}{|l|}{$\mathrm{pT}, n(\%)$} \\
\hline 1 & $26(29.88)$ \\
\hline 2 & $27(31.03)$ \\
\hline 3 & $7(8.05)$ \\
\hline 4 & $4(4.60)$ \\
\hline$x$ & $23(26.44)$ \\
\hline \multicolumn{2}{|l|}{$\mathrm{pN}, n(\%)$} \\
\hline 0 & $18(20.69)$ \\
\hline 1 & $27(31.03)$ \\
\hline 2 & $10(11.49)$ \\
\hline 3 & $10(11.49)$ \\
\hline$x$ & $22(25.30)$ \\
\hline \multicolumn{2}{|l|}{ HR status, $n(\%)$} \\
\hline $\mathrm{ER}+/ \mathrm{PgR}+$ & $59(67.81)$ \\
\hline $\mathrm{ER}+/ \mathrm{PgR}-$ & $24(27.59)$ \\
\hline $\mathrm{ER}-/ \mathrm{PgR}+$ & $0(0.0)$ \\
\hline $\mathrm{ER}-/ \mathrm{PgR}-$ & $4(4.60)$ \\
\hline Median (range) \%ER + staining & $70(20-100)$ \\
\hline Median (range) $\% \mathrm{PgR}+$ staining & $50(10-80)$ \\
\hline \multicolumn{2}{|l|}{ HER2 status, $n(\%)$} \\
\hline Positive & $82(94.25)$ \\
\hline Negative & $4(4.60)$ \\
\hline Unknown & $1(1.15)$ \\
\hline \multicolumn{2}{|l|}{ Adjuvant CT, $n(\%)$} \\
\hline Yes & $45(51.72)$ \\
\hline No & $36(41.38)$ \\
\hline Unknown & $6(6.90)$ \\
\hline \multicolumn{2}{|l|}{ Adjuvant trastuzumab, $n(\%)$} \\
\hline Yes & $25(28.73)$ \\
\hline No & $54(62.07)$ \\
\hline Unknown & $8(9.20)$ \\
\hline \multicolumn{2}{|l|}{ Adjuvant ET, $n(\%)$} \\
\hline Yes & $52(59.77)$ \\
\hline No & $29(33.33)$ \\
\hline Unknown & $6(6.90)$ \\
\hline \multicolumn{2}{|l|}{ Metastatic sites } \\
\hline Bone-only & $15(17.24)$ \\
\hline Visceral & $50(57.47)$ \\
\hline Other & $22(25.29)$ \\
\hline \multicolumn{2}{|l|}{ Prior lines of therapy CT, $n(\%)$} \\
\hline 0 & $23(26.44)$ \\
\hline 1 & $20(22.99)$ \\
\hline 2 & $15(17.24)$ \\
\hline$\geq 3$ & $29(33.33)$ \\
\hline
\end{tabular}


Table 1 (continued)

\begin{tabular}{ll}
\hline Median age, years (range) & $63(35-87)$ \\
\hline Prior lines of therapy ET, $n(\%)$ & \\
0 & $16(18.39)$ \\
1 & $51(58.62)$ \\
2 & $17(19.55)$ \\
3 & $3(3.44)$ \\
Last treatment before F+T, $n(\%)$ & \\
CT + trastuzumab & $23(26.43)$ \\
ET + trastuzumab & $20(22.99)$ \\
T DM1 & $8(9.20)$ \\
Lapatinib +capecitabine & $4(4.60)$ \\
CT & $12(13.79)$ \\
Trastuzumab & $1(1.15)$ \\
ET & $10(11.49)$ \\
Clinical trial & $1(1.15)$ \\
Unknown & $8(9.20)$ \\
\hline
\end{tabular}

$H R$ hormone receptor, $E R$ estrogen receptor, $P g R$ progesterone receptor, $C T$ chemotherapy, ET endocrine therapy, $F$ fulvestrant, $T$ trastuzumab

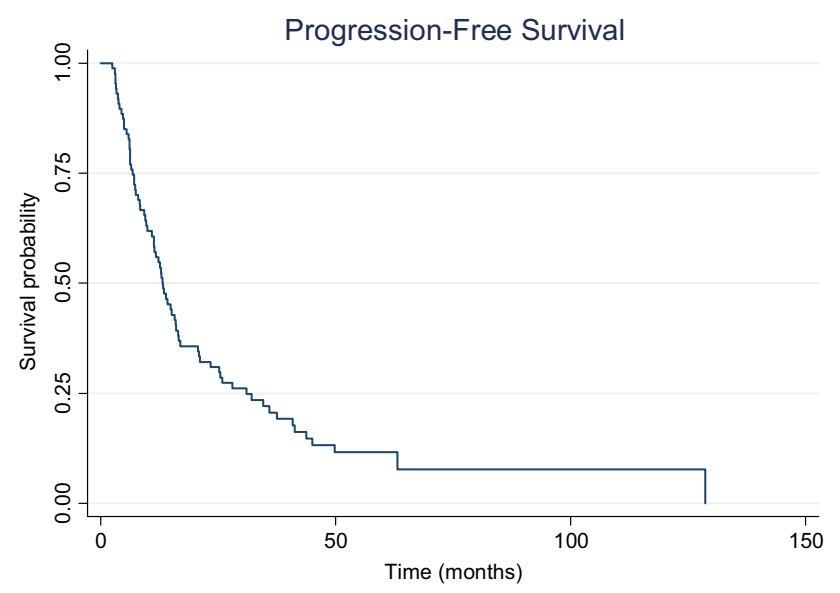

Fig. 1 Progression-free survival in the overall population

progression to a previous line or as maintenance (mPFS 12.9 and 13.9 months, respectively; $p=0.67$ ), neither considering the number of previous treatment lines $(\leq 3$ or $<3)$ $(p=0.34)$, the type of previous therapy (chemotherapy or endocrine therapy), or triple positive vs single HR expression overall $(p=0.22)$.

\section{Discussion}

In the era of tailored treatments the most appropriate therapy of $\mathrm{HR}+/ \mathrm{HER} 2+\mathrm{BC}$ is a matter of debate. While showing less responsiveness to the combination of chemotherapy plus anti-HER2 agents as compared to the HR negative counterpart, results with the combination of anti-HER2 agents and non-steroidal aromatase inhibitors (NSAI) have been quite disappointing with a mPFS not exceeding 4.8 months with trastuzumab and anastrozole and 8.2 months with lapatinib and letrozole as first-line treatment $[6,7]$. The recently updated ALTERNATIVE trial, which investigated the addition of lapatinib, trastuzumab, or both agents to a NSAI, showed a significant improvement in mPFS for the double HER2 blockade vs trastuzumab plus NSAI (11 vs 5.6 months) [15]. On the other hand the PERTAIN trial reported a mPFS of 21.7 months in the arm with the double HER2 blockade with pertuzumab and trastuzumab plus a NSAI, which was not only significantly higher than that reported by the trastuzumab plus NSAI combination but, more importantly, was similar to that reported in patients who had received chemotherapy before endocrine therapy (mPFS $=18.9$ months) [16]. The relative small number of patients (54) and the non-randomized assignment to the chemotherapy/no chemotherapy arms leading to a smaller proportion of patients with poor prognostic features in the latter arm, do not allow to qualify the chemotherapy-free combination as a standard first-line treatment for $\mathrm{HR}+1$ HER 2 + ABC, but clearly represents a strong rationale for further studies to identify which patients may be spared unnecessary chemotherapy [16].

Fulvestrant was proven superior to NSAI in patients with HR +/HER2-ABC [17] and has been considered as favorite anti-ER partner in combination with other targeted therapies in HR +/HER2 + breast cancer $(\mathrm{Na}-\mathrm{PHer} 2$, MonarcHER), despite the lack of clinical data of the activity of this combination [18, 19]. Fulvestrant monotherapy showed an encouraging activity in a retrospective study in 102 pretreated patients with $\mathrm{HR}+/ \mathrm{HER} 2+\mathrm{ABC}$ with a $\mathrm{CBR}$ of $42 \%$ and a median duration of treatment of 8.1 months, which raised to 14.5 months in patients achieving clinical benefit. Among them only 5 patients had received concurrent trastuzumab [20]. Bartsch et al. reported outcomes of 112 patients with $\mathrm{HR}+$ tumors treated with Fulvestrant 20 of whom had HR +/HER $2+$ tumors and HER2 status did not predict treatment efficacy [21]. Rusz et al. reported a durable benefit (12, 25, and 28 months) with Fulvestrant monotherapy in 3 pretreated patients with triple-positive ABC [22]. The SystHER, an observational prospective registry study reporting tumor characteristics, treatment patterns, and outcome of patients with HER $2+\mathrm{ABC}$ treated in the USA from 2012 to 2016, included 46 patients who received Fulvestrant but no further information on the setting and outcome is available yet [23].

To our knowledge this is the first report of the combination of fulvestrant + trastuzumab in a clinically relevant number of patients. We observed a mPFS of 12.9 months and a CBR of $78 \%$ which favorably compare with those previously reported by the combination of a NSAI and trastuzumab 
even when administered in earlier lines $[6,7,15,16]$. Only the addition of pertuzumab gained a superior MPFS in the PERTAIN study [16]. As expected, our results favorably compare also with those obtained with single agent Fulvestrant, particularly in terms of CBR [20-22].

Remarkably, $77 \%$ of our patients had been treated with $\geq 3$ previous therapies for metastatic disease, $73 \%$ started fulvestrant + trastuzumab after progression to a previous regimen, and more than $50 \%$ had visceral disease. The short TTP (7.5 months) observed with the treatment preceding the fulvestrant + trastuzumab combination confirms that our unselected population represented a really poor prognosis cohort.

Fulvestrant was administered as first-line endocrine therapy only in $19 \%$ of patients but its activity did not seem to be affected by prior endocrine resistance since the patients (20) who had progressed on endocrine therapy + trastuzumab had a mPFS of 14.26 months (4.37-119.93), thus maintaining responsiveness to a different endocrine manipulation. On the other hand, also patients progressing after a chemotherapy containing regimen experienced an mPFS of 12.73 months (range 3.07-59.57); in both cohorts the mPFS was significantly longer than the TTP obtained with the preceding therapy underlining the activity of the combination irrespective of prior treatments.

Despite the benefit of endocrine maintenance therapy has not been formally investigated in a randomized trial, evidence from PERTAIN and real-world reports support the efficacy of a sustained blockade of the ER signaling in HR +/HER 2 + breast cancer $[25,26]$. Our results reporting a mPFS of 13.9 months in patients who received maintenance fulvestrant are in line with these reports.

Interestingly, in our study triple-positive tumors did not fare better than single HR-positive tumors. Triple-positive breast cancer, which accounts for $5-10 \%$ of all breast cancers, might represent a distinct subtype among luminal HER2 breast cancer with more exquisite endocrine sensitiveness. [27]. However, triple-positive breast cancer has been described as a heterogeneous population. A genomic analysis of 5 cohorts of triple-positive breast cancer showed lower levels of HER 2 mRNA and protein expression than ER and PgR positive/HER2 + breast cancers. More than $40 \%$ of triple-positive breast cancers were classified as the luminal A intrinsic subtype, with an even lower HER2 expression level and were associated with a better prognosis and a reduced benefit from trastuzumab [28]

In a large retrospective series of 872 patients Vici et al. showed a significant interaction between HR expression and trastuzumab benefit since patients with triple positive tumors and $\mathrm{HR}>50 \%$ gained a modest benefit from adjuvant antiHer2 treatment [29]. In our series ER status maintained a predictive role, while PgR was not associated with response. The loss of PgR, which may be also attributable to previous endocrine treatment, apparently did not represent a feature of endocrine resistance, as suggested by its lower expression in metastatic sites without affecting treatment efficacy.

A reduced benefit of the combination of chemotherapy and trastuzumab in the adjuvant setting was shown in a retrospective analysis of the National Cancer Database among 37777 stage I luminal HER2 + breast cancer. A large proportion had received only the combination of endocrine therapy and trastuzumab and had an improved overall survival as compared with patients who were treated with chemotherapy and trastuzumab [30].

Fulvestrant has been chosen as preferred endocrine partner in combination with anti-HER2 agents and CDK4/6 inhibitors either in the early and advanced setting [18, 19], In the phase II Na-PHER2 study the combination of pertuzumab, trastuzumab, fulvestrant, and palbociclib achieved an objective response and a pathological complete response in 97 and $27 \%$ of patients with large HR + /HER 2 + tumors, respectively [18].

The MonarcHER study randomized the combination of fulvestrant, trastuzumab and abemaciclib vs trastuzumab, and abemaciclib vs trastuzumab plus chemotherapy in heavily pretreated HR $+/ \mathrm{HER} 2+\mathrm{ABC}$ [19]. The triple targeted regimen resulted in a mPFS of 8.3 months significantly higher than that obtained with chemotherapy, suggesting a benefit of the addition of the blockade of the CDK4/6 pathway. The lack of an arm containing fulvestrant and trastuzumab has represented the major criticism to these results since this did not allow to evaluate the net benefit of the CDK4/6 inhibitor.

These results, along with other indirect evidence, suggest that at least a proportion of luminal HER 2 + breast cancer may experience a favorable outcome without chemotherapy.

We are aware of several limitations of our real-world study as compared with prospective trials. First of all the retrospective design which may have not allowed to meet with a strict application of RECIST 1.1 criteria in all the patients. In addition the majority of our patients had not received pertuzumab in earlier lines and this may have led to the inclusion of a relatively less resistant cohort. Finally, given the multicentric design, the sequence of treatments varied among the different Hospitals and might have interfered with the results obtained especially with the definition of predictive factors of efficacy.

Despite these limitations, our results provide a piece of evidence in the refinement of the optimal treatment of $\mathrm{HR}+/ \mathrm{HER} 2+$ breast cancer. Our results suggest that the combination of fulvestrant and trastuzumab is active and may deserve further investigations, possibly in combination with pertuzumab and in patients with high ER expression. Patients with de novo HR +/HER2 + ABC, who represent $30 \%$ to $50 \%$ of patients receiving first-line treatment, generally experience better outcome as compared with those 
with recurrent $\mathrm{ABC}$ [24]. For instance, it would be interesting to test this combination \pm CDK $4 / 6$ inhibitors vs the chemotherapy containing triplet in this population at better prognosis to understand whether the algorithm of treatment of HER2 + breast cancer may be tailored according to the HR status.

Acknowledgements The authors are indebted with Dr Elisa Crotti for her invaluable support in the realization of the study.

Author contributions All authors contributed to the study conception and design. Material preparation, data collection, and analysis were performed by RT, RP, RdS, PV, GB, LC, VL, RG, AF, MRV, ARG, $\mathrm{BT}$, and LP. The first draft of the manuscript was written by RT and all authors revised all the versions of the manuscript. All authors read and approved the final manuscript.

Funding The authors did not receive any financial support.

Data availability The datasets generated during and analyzed during the current study are available from the corresponding author on reasonable request.

\section{Declarations}

Conflict of interest Rosalba Torrisi: Astra Zeneca, Eisai, Pfizer, Eli Lilly, and Gentili. Rita De Sanctis Novartis, Amgen, Kyowa Kirin, and Eisai. Patrizia Vici: Roche, Pfizer, Novartis, Gentili, Eli Lilly, and Eisai. Giulia Valeria Bianchi: Eli Lilly and Novartis. Laura Cortesi: Novartis, Amgen, Astra Zeneca, and Pfizer. Laura Pizzuti Roche, Pfizer, Novartis, Gentili, and Eisai. Marina Elena Cazzaniga: Astra Zeneca, Novartis, Eli Lilly, Eisai Pierre Fabre, and Roche. Armando Santoro BMS, Servier, Gilead, Pfizer, Eisai, Bayer, MSD, Takeda, Roche, Astra Zeneca, Pfizer, Eli Lilly, Novartis, Aqule, Sandoz, and Abb-Vie.

Ethical approval The study was approved by the Ethical Committees of all the participating Institutions. The study was conducted in compliance with Helsinki Declaration.

Consent to participate All patients provided written consent for the use of their anonymized clinical data for scientific purpose.

\section{References}

1. Ferrari A, Vincent-Salomon A, Pivot X, Sertier AS, Thomas E, Tonon L et al (2016) A whole-genome sequence and transcriptome perspective on HER2-positive breast cancers. Nat Commun 7:12222. https://doi.org/10.1038/ncomms 12222

2. Mehta A, Tripathy D (2014) Co-targeting estrogen receptor and HER2 pathways in breast cancer. The Breast 23:2-9. https://doi. org/10.1016/j.breast.2013.09.006

3. Prat A, Carey LA, Adamo B, Vidal M, Tabernero J, Cortés J et al (2014) Molecular Features and Survival Outcomes of the Intrinsic Subtypes Within HER2-Positive Breast Cancer. J Natl Cancer Inst 106:dju152. https://doi.org/10.1093/jnci/dju152

4. Cardoso F, Paluch-Shimon S, Senkus E, Curigliano G, Aapro MS, André F et al (2020) 5th ESO-ESMO international consensus guidelines for advanced breast cancer (ABC 5). Ann Oncol 31:1623-1649. https://doi.org/10.1016/j.annonc.2020.09.010
5. Osborne CK, Schiff R (2011) Mechanisms of endocrine resistance in breast cancer. Annu Rev Med 62:233-247. https://doi.org/10. 1146/annurev-med-070909-182917

6. Kaufman B, Mackey JR, Clemens MR, Bapsy PP, Vaid A, Wardley A et al (2009) Trastuzumab plus anastrozole versus anastrozole alone for the treatment of postmenopausal women with human epidermal growth factor receptor 2-positive, hormone receptor-positive metastatic breast cancer: Results from the randomized phase III TAnDEM study. J Clin Oncol 27:5529-5537. https://doi.org/10.1200/JCO.2008.20.6847

7. Johnston S, Pippen J Jr, Pivot X, Lichinitser M, Sadeghi S, Dieras $V$ et al (2009) Lapatinib combined with letrozole versus letrozole and placebo as first-line therapy for postmenopausal hormone receptor-positive metastatic breast cancer. J Clin Oncol 27:5538 5546. https://doi.org/10.1200/JCO.2009.23.3734

8. von Minckwitz G, Untch M, Blohmer J-U, Costa SD, Eidtmann H, Fasching PA et al (2012) Definition and impact of pathologic complete response on prognosis after neoadjuvant chemotherapy in various intrinsic breast cancer subtypes. J Clin Oncol 30:17961804. https://doi.org/10.1200/JCO.2011.38.8595

9. Abramovitz M, Williams C, Loibl S et al (2016) Dual blockade of HER-2 provides a greater magnitude of benefit in patients with hormone-negative versus hormone positive breast cancer. Clin Breast Cancer 16:444-455. https://doi.org/10.1016/j.clbc.2016. 06.004

10. Cortazar P, Zhang L, Untch M, Mehta K, Costantino JP, Wolmark $\mathrm{N}$ et al (2014) Pathological complete response and long-term clinical benefit in breast cancer: the CTNeoBC pooled analysis. Lancet 384:164-172. https://doi.org/10.1016/S0140-6736(13) $62422-8$

11. Montemurro F, Rossi V, Cossu Rocca M, Martinello R, Verri E, Redana $S$ et al (2012) Hormone receptor expression and activity of trastuzumab with chemotherapy in HER2-positive advanced breast cancer patients. Cancer 118:17-26. https://doi.org/10.1002/ cncr.26162

12. Croessmann S, Formisano L, Kinch LN, Gonzalez-Ericsson PI, Sudhan DR, Nagy RJ et al (2019) Combined blockade of activating ERBB2 mutations and ER results in synthetic lethality of ERp/ HER2 Mutant Breast Cancer. Clin Cancer Res 25(1):277-289. https://doi.org/10.1158/1078-0432

13. Robertson JFR (2007) Fulvestrant (faslodex) - how to make a good drug better. Oncologist 12:774-784. https://doi.org/10.1634/ theoncologist.12-7-774

14. Chen Q, Weng Z, Lu Y, Jia Y, Ding L, Bai F et al (2017) An experimental analysis of the molecular effects of trastuzumab (herceptin) and fulvestrant (falsodex), as single agents or in combination, on human HR+/HER2+ breast cancer cell lines and mouse tumor xenografts. PLoS ONE 12:e0168960. https://doi. org/10.1371/journal.pone.0168960

15. Johnston SR, Egg R, Im S-A, Park IH, Burdaeva O, Kurteva G et al (2020) Phase III, randomized study of dual human epidermal growth factor receptor 2 (HER2) blockade with lapatinib plus trastuzumab in combination with an aromatase inhibitor in postmenopausal women with HER2-positive, hormone receptor-positive metastatic breast cancer: updated results of alternative. J Clin Oncol 36(8):741-748

16. Rimawi M, Ferrero J-M, de la Haba-Rodriguez J, Poole C, De Placido S, Osborne CK et al (2018) First-line trastuzumab plus an aromatase inhibitor, with or without pertuzumab, in human epidermal growth factor receptor 2-positive and hormone receptorpositive metastatic or locally advanced breast cancer (PERTAIN): a randomized, open-label phase II trial. J Clin Oncol 36:28262835. https://doi.org/10.1200/JCO.2017.76.7863

17. Robertson JFR, Bondarenko IM, Trishkina E, Dvorkin M, Panasci L, Manikhas A et al (2016) Fulvestrant 500 mg versus anastrozole $1 \mathrm{mg}$ for hormone receptor-positive advanced breast cancer 
(FALCON): an international, randomised, double-blind, phase 3 trial. Lancet 388:2997-3005. https://doi.org/10.1016/S01406736(16)32389-3

18. Gianni L, Bisagni G, Colleoni M, Del Mastro L, Zamagni C, Mansutti $\mathrm{M}$ et al (2018) Neoadjuvant treatment with trastuzumab and pertuzumab plus palbociclib and fulvestrant in HER2-positive, ER-positive breast cancer. Lancet Oncol 19:249-256. https://doi. org/10.1016/S1470-2045(18)30001

19. Tolaney SM, Wardley AM, Zambelli S, Hilton JF, Troso-Sandoval TA, Ricci F et al (2020) Abemaciclib plus trastuzumab with or without fulvestrant versus trastuzumab plus standard-of-care chemotherapy in women with hormone receptor-positive, HER2positive advanced breast cancer (monarcHER): a randomised, open-label, phase 2 trial. Lancet Oncol 21:763-775. https://doi. org/10.1200/JCO.20.02514

20. Robertson JFR, Steger GG, Neven P, Barni S, Gieseking F, Nole F et al (2010) Activity of fulvestrant in HER2-overexpressing advanced breast cancer. Ann Oncol 21:1246-1253. https://doi. org/10.1093/annonc/mdp447

21. Bartsch R, Wenzel C, Altorjai G, Pluschnig U, Mader RM, Gnant $M$ et al (2007) Her2 and progesterone receptor status are not predictive of response to fulvestrant treatment. Clin Cancer Res 13:44359. https://doi.org/10.1158/1078-0432.CCR-06-3050

22. Rusz O, Koszo E, Dobi A, Valicsek E, Nikoleny A, Uhercsak $\mathrm{G}$ et al (2018) Clinical benefit of fulvestrant monotherapy in the multimodal treatment of hormone receptor and HER2 positive advanced breast cancer: a case series. Onco Targets Ther 11:5459-5463. https://doi.org/10.2147/OTT.S170736

23. Cobleigh M, Yardley DA, Brufsky AM, Rugo HS, Swain SM, Kaufman PA et al (2020) Baseline characteristics, treatment patterns, and outcomes in patients with HER2-positive metastatic breast cancer by hormone receptor status from SystHERs. Clin Cancer Res 26:1105-1113. https://doi.org/10.1158/1078-0432. CCR-19-2350

24. Tripathy D, Kaufman PA, Brufsky AM, Mayer M, Yood MU, Yoo B et al (2013) First line treatment patterns and clinical outcomes in patients with HER2-positive and hormone receptor-positive metastatic breast cancer from registher. Oncologist 18:501-510. https://doi.org/10.1634/theoncologist.2012-0414

25. Gamucci T, Pizzuti L, Natoli C, Sperduti ML, Barba I et al (2019) A multicenter retrospective observational study of first-line treatment with pertuzumab, trastuzumab and taxanes for advanced HER2 positive breast cancer patients. Reper study. Cancer Biol Ther 20:192-200. https://doi.org/10.1080/15384047.2018.15230 95

26. Vici P, Pizzuti L, Natoli C, Gamucci T, Di Lauro L, Barba M et al (2015) Triple positive breast cancer: a distinct subtype? Cancer Treat Rev 41:69-76. https://doi.org/10.1016/j.ctrv.2014.12.005

27. Zhao S, Liu X-Y, Jin X, Ma D, Xiao Y, Shao Z-M, Jiang Y-Z (2019) Molecular portraits and trastuzumab responsiveness of estrogen receptor-positive, progesterone receptor-positive, and HER2-positive breast cancer. Theranostics 9:4935-4945. https:// doi.org/10.7150/thno. 35730

28. Vici P, Pizzuti L, Sperduti I, Frassoldati A, Natoli C, Gamucci $T$ et al (2016) Triple positive early breast cancer: an observational multicenter retrospective analysis of out come. Oncotarget 7:17932-17944. https://doi.org/10.18632/oncotarget.7480

29. Nahleh ZA, Elimimian EB, Elson LC, Hobbs B, Wei W, Blake CN (2020) Endocrine therapy plus anti-HER2 therapy as adjuvant systemic therapy for luminal HER2-positive breast cancer: an analysis of the national cancer database. Breast Cancer 14(1):12. https://doi.org/10.1177/1178223420945694

30. Swain SM, Baselga J, Kim SB, Ro J, Semiglazov V, Campone M et al (2015) Pertuzumab, trastuzumab, and docetaxel in HER2positive metastatic breast cancer. N Engl J Med 372:724-734. https://doi.org/10.1056/NEJMoa1413513

Publisher's Note Springer Nature remains neutral with regard to jurisdictional claims in published maps and institutional affiliations. 